\title{
A PRÁtiCA dA LAVAGEM dAS MÃOS PELA EQUIPE DE ENFERMAGEM DE UMA MATERNIDADE DE CARUARU-PE
}

\author{
Nayale Lucinda Andrade ALBUQUERQUE * \\ Crislayne Thaise da SILVA ** \\ Érica Priscila Barcelos dos SANTOS *** \\ Luiz Fernando de Andrade SILVA ****
}

Recebido em: 16/09/2015 - Aprovado em: 21/05/2016 - Disponibilizado em: 30/07/2016

\begin{abstract}
RESUMO
Objetivo: foi avaliar a prática da lavagem das mãos pela equipe de enfermagem de uma maternidade de Caruaru- Pe. Método: a pesquisa foi realizada com 63 profissionais, destes 16 enfermeiros e 47 técnicos que atuam na triagem obstétrica, pré parto, pré eclampsia e alojamento conjunto. Os dados foram obtidos através de uma entrevista estruturada e pelo método observacional utilizando um instrumento de check-list com passos da técnica.Resultados: Os profissionais enfermeiros obtiveram um melhor desempenho, quanto a fricção do polegar com estatística significante de $\mathrm{p}(0,0003)$ quando comparados aos técnicos. No que se refere ao tempo da técnica, todos (100\%) obtiveram tempo superior ao preconizado (15 segundos). Conclusão: Apesar da disponibilidade dos produtos para a lavagem das mãos e a existência de cartazes divulgando as etapas da técnica, os profissionais não realizaram o procedimento conforme as recomendações, evidenciando a necessidade da implementação de atividades educativas para intensificar a adesão dos profissionais.

Descritores: Lavagem de Mãos; Infecção Hospitalar; Educação em Saúde.
\end{abstract}

\section{THE PRACTICE OF HANDWASHING BY NURSING STAFF OF A}

\section{MATERNITY LEAVE OF CARUARU-PE}

\begin{abstract}
The purpose of this study was to evaluate the practice of hand washing by the nursing staff of a maternity hospital in Caruaru. The survey was conducted with 63professionals, of these 16 nurses and 47 technicians who work in the obstetric triage, pre birth, pre-eclampsia and rooming. Data were obtained through a structured interview and
\end{abstract}

* Enfermeira Especialista em Saúde da Mulher pelo programa de residência do Instituto Materno Infantil de Pernambuco, IMIP ; Mestre em Ciências da Saúde pela Universidade Federal de Pernambuco, UFPE;Professora do Curso de Graduação em Enfermagem da Associação Caruaruense de Ensino Superior, ASCES;e-mail: nayalelucinda@gmail.com

** Enfermeira Graduada em Enfermagem pela Associação Caruaruense de Ensino Superior, ASCES; Pos graduanda em Unidade de Terapia Intensiva,e-mail: crislayne tds@ hotmail.com;

*** Enfermeira Graduada em Enfermagem pela Associação Caruaruense de Ensino Superior, ASCES , Pos graduanda em Nefrologia pela Faculdade de Enfermagem Nossa Senhora das Graças da Universidade de Pernambuco, email: ericapriscila@hotmail.com;

**** Enfermeiro Graduado em Enfermagem pela Associação Caruaruense de Ensino Superior, ASCES; Residente de Enfermagem em Pneumologia pelo Hospital Otávio de Freitas, Recife-PE, email: luizfernando989@gmail.com 
the observational method using an instrument with a checklist of technicalsteps. The nurses had a better performance, as the friction of the thumb with a statistically significant $p(0.0003)$ when compared to technicians. With regard to the time of the technique, all (100\%) obtained longer than recommended (15 seconds).Despite the availability of products for handwashing and posters publicizing theexistence of stages of the technique, the professionals did not perform the procedureaccording to the recommendations, highlighting the need to implement educational activities to enhance the adhesion of professionals.

Keywords: Handwashing;Hospital Infection; Health Education

\section{INTRODUÇÃO}

As infecções hospitalares ocorrem por diversas razões e existem muitos mecanismos que favorecem seu aparecimento, um desses é a transmissão de microorganismos pelos profissionais da área de saúde, que atuam como vetores, direta ou indiretamente. Ou seja, a infecção hospitalar é definida como aquela adquirida após a internação do paciente e que se manifesta durante a internação ou mesmo após a alta. (PEREIRA, 2005). De acordo com Veronesi ( 2005), esta é relacionada com a assistência dos profissionais de saúde, sendo causada por um desequilíbrio da relação existente entre a microbiota humana normal e os mecanismos de defesa do hospedeiro.

Segundo Paulo (2008), a aquisição de microrganismos ocorre, geralmente, a partir da transmissão pelo contato das mãos dos profissionais com os pacientes e pelo contato direto do paciente com material ou ambiente contaminado. Visto em Brunner e Suddart (2005) que a lavagem das mãos (LM) reduz a quantidade da flora normal benigna e as bactérias transitórias, diminuindo o risco de transferência para os pacientes.
A ANVISA (2007) relata que aproximadamente 40 milhões de hospitalizações por ano nos EUA, estima-se que 2 milhões de pacientes, ou seja, 5\% do total de hospitalizações, adquirem IH.

Paulo (2008) reforça que, a infecção hospitalar (IH) é considerada um importante problema de saúde pública, por contribuir com o aumento na morbidade, mortalidade, tempo de internação e gastos com procedimentos diagnósticos e terapêuticos.

No século XIX, Ignaz Philipp Semmelweis constatou a importância da transmissão cruzada. Este instituiu como método eficaz de controle de disseminação de doenças a obrigatoriedade da lavagem das mãos antes do início do parto e no atendimento a toda e qualquer paciente após o parto (PAULO, 2008).

Sendo assim o Ministério da saúde criou o Programa de Controle de Infecções Hospitalares (PCIH) com finalidade de reduzir ao máximo a incidência e a gravidade das infecções hospitalares. Dentre as ações propostas, destaca-se o papel da higienização das mãos como ação mais importante na prevenção e controle das infecções em serviços de saúde (ANVISA,2007). 
A partir das literaturas é observado que apesar da lavagem das mãos ser um procedimento simples, de baixo custo $\mathrm{e}$ eficiente no controle da infecção hospitalar, nota-se pouca adesão por parte dos profissionais de saúde, como também é observado desconhecimento da técnica correta (MARTINEZ, 2009; SURTON, 2005).

Diante do exposto, percebeu-se a necessidade de realizar um estudo sobre o assunto na cidade de Caruaru-PE, onde há uma escassez de publicações nessa área, com intuito de avaliar a prática da lavagem das mãos pelos profissionais de Enfermagem. Essa avaliação possibilitará um diagnóstico situacional, e beneficiará toda população assistida na instituição de saúde, pois subsidiará os profissionais de enfermagem para o enfrentamento da $\mathrm{IH}$, como também servirá para o aperfeiçoamento técnico científico da equipe de enfermagem.

\section{OBJETIVOS}

Verificar o perfil sócio demográfico dos profissionais de enfermagem.

Comparar a execução da prática de lavagem das mãos entre os profissionais enfermeiros, técnicos de enfermagem e auxiliares de enfermagem.

Verificar o grau de adesão à técnica de lavagem das mãos entre os profissionais enfermeiros, técnicos de enfermagem e auxiliares de enfermagem.
Identificar o tempo gasto por cada profissional de enfermagem para lavagem das mãos.

\section{FUNDAMENTAÇÃO TEÓRICA}

Segundo Scheidt (2006), a lavagem das mãos é tradicionalmente, o ato mais importante para a prevenção e o controle das infecções hospitalares. Há pelo menos 150 anos, sua importância foi comprovada por Semmelweis, ao introduzi-la, antes do atendimento a partos, sendo observada a redução das taxas de infecção puerperal (PEREIRA, 2005).

Paulo (2008), demosntrou que a higienização das mãos visa a reduzir a transmissão de microrganismos, prevenindo infecções sendo necessário realizar antes e depois de qualquer procedimento, seja ele invasivo ou não. De acordo com Santos (2009), esta é considerada uma prática de assepsia simples que continua sendo a principal forma de prevenir e controlar as infecções, além de gerar benefícios extensíveis aqueles envolvidos no processo de cuidado, devendo configurar-se como um hábito de todos os profissionais de saúde.

O Ministério da saúde ressalta que os benefícios destas práticas são inquestionáveis, desde a redução da morbidade e mortalidade dos pacientes até a diminuição de custos associados ao tratamento dos quadros infecciosos (ANVISA, 2007). 
Em 1989, o Ministério da Saúde (MS) do Brasil editou o manual "Lavar as mãos" com o objetivo de normatizar essa técnica nas unidades de saúde brasileiras, proporcionando aos profissionais de saúde subsídios técnicos relativos às normas e aos procedimentos para lavar as mãos, visando assim a prevenção das infecções hospitalares (SANTOS, 2009).

A importância dessa prática continuou sendo reconhecida pelo MS, quando esse incluiu recomendações para a higiene das mãos na portaria 2616/98, de 12 de maio de 1998 (FELIX, 2009). Segundo a Portaria $n^{\circ}$ 2.616 de 1998, o Ministério da saúde criou o Programa de Controle de Infecções Hospitalares (PCIH) com finalidade de reduzir ao máximo a incidência e a gravidade destas infecções. E para sua execução, as instituições de saúde passaram a conter uma Comissão de Controle de Infecção Hospitalar (CCIH) que é o órgão ligado à autoridade máxima da instituição que executa as ações de controle de IH. Dentre as ações propostas, destaca-se o papel da higienização das mãos na portaria 2616/98, de 12 de maio de 1998 (ANVISA, 2007).

Conforme Paulo (2008) demonstra, apesar de mais de um século e meio depois da instituição da lavagem das mãos, ainda verifica-se uma baixa adesão a ela na rotina diária dos profissionais da saúde. Visto que equipe multiprofissional normalmente apresenta também um grande volume de atividades a serem realizadas.
Observa-se em Pereira (2005) que não é incomum a realização da técnica de $\mathrm{LM}$ de forma rápida e desatenta, o que pode aumentar a incidência no quadro de infecções hospitalares, principalmente para os profissionais que prestam atendimento a pacientes mais vulneráveis.

Ximenes (2007) ressalta que a conduta do enfermeiro deve ser firme e cuidadosa, devendo assegurar às gestantes e à equipe de profissionais a proteção desejada durante a assistência ao trabalho de parto e parto, contra a contaminação cruzada e a transmissão de doenças infecciosas.

Para a maioria das mulheres, a maternidade é um momento especial em suas vidas. Momento que, quando acompanhado de cuidado humanizado, e atitudes sensíveis da equipe de saúde, constituída por profissionais conscientes do papel que desempenham, proporciona segurança e bemestar (CARDOSO, 2008).

Segundo Martini (2004), nas maternidades, os profissionais de saúde e as mães manuseiam o recém-nascido diariamente, devendo-se considerar que estes são desprovidos de imunidade e de microbiota própria. Para Palos (2009) estas condições os colocam mais vulneráveis às infecções hospitalares, o que mostra a importância de os profissionais e das mães manterem suas mãos higienizadas.

Percebe-se a grande necessidade de modificações profundas na qualidade da 
assistência executada nas maternidades brasileiras (XIMENES, 2007). A humanização da assistência ao parto contempla toda uma mudança na lógica assistencial e na prática profissional, como também a disposição de material, de equipamentos e de estrutura física apropriada e adequada às necessidades da gestante (PEREIRA, 2005).

De acordo com Paulo (2008), a preparação da sala de parto varia, mas alguns princípios devem ser seguidos, por tratar se de pré-requisitos de suma importância como, por exemplo, realização de assepsia, e antissepsia, com o intuito de reduzir os riscos de infecção na mãe e no recém-nascido. Ximenes (2007), ressalta que o enfermeiro torna-se $o$ profissional de ponta na efetivação das medidas de biossegurança.

Programas educacionais direcionados à prática da lavagem das mãos apresentam, em sua maioria, resultados limitados onde as intervenções educativas podem influenciar o comportamento adequado destes profissionais (SHEIDT, 2006).

Segundo Carraro (2008), o enfermeiro é considerado um elemento essencial nos serviços de saúde por ter a capacidade de articular as diversas ações propostas pela equipe multidisciplinar, devendo assim estar diretamente envolvido no planejamento de programas de educação permanente nas instituições de saúde.
Diante desse contexto é fundamental que os profissionais compreendam as medidas preventivas em saúde e que possam contribuir para a elaboração de estratégias inovadoras na educação permanente dos profissionais desta área, tendo como alvo principal o próprio profissional. Sendo necessário entender a importância da lavagem das mãos e a complexidade que a realização incorreta ou a não realização da mesma traz como implicações para as instituições, profissionais e paciente.

\section{MÉTODO}

$\mathrm{O}$ estudo foi desenvolvido e fundamentado em uma abordagem quantitativa descritiva do tipo transversal. Realizado na Maternidade Jesus Nazareno no Município de Caruaru-Pe, por ser um serviço de referência em assistência materno-infantil de alto risco para Caruaru e regiões circunvizinhas. Os setores escolhidos para a pesquisa foram aqueles os quais há uma assistência mais intensa à mulher, ou seja, maior contato físico com a cliente, portanto, a triagem, sala de pré-parto, pós-parto e sala de pré-eclâmpsia.

Existem áreas para lavagem das mãos e escovação para cada setor, com o material necessário a este procedimento (clorexidina degermante e papel toalha).

A população foi composta por todos os profissionais de enfermagem durante a realização da LM. É importante salientar que 
todos os profissionais, que concordaram em participar da pesquisa, receberam informações sobre o interesse, a justificativa, o objetivo, os riscos e benefícios e as finalidades do estudo mediante a apresentação do Termo de Consentimento Livre e Esclarecido (TCLE), seguindo as determinações da Resolução 196/96 do Conselho Nacional de Saúde (2005), que trata dos aspectos éticos da pesquisa envolvendo seres humanos.

Para a coleta dos dados, o instrumento, elaborado pelos autores, foi construído em 02 partes.

A etapa I, é uma entrevista estruturada composta por dados sóciodemográficos e questões sobre os conhecimentos da biossegurança relacionados à lavagem das mãos, onde os profissionais escolheram apenas 01 alternativa.

A etapa II, é um instrumento com os passos da técnica de lavagem das mãos, baseado na recomendação do Ministério da Saúde (ANVISA, 2007), na forma de lista de checagem ou Check List, no qual o observador aponta uma das 3 alternativas de categorização: sim (desempenho adequado), não (desempenho inadequado) ou parcialmente em cada ação desempenhada pelos profissionais de enfermagem. Ao final deste instrumento, contém um espaço para inserção do tempo gasto por cada profissional de enfermagem para lavagem das mãos.

Os itens checados na etapa II são, em ordem de prioridades: o primeiro passo a retirada dos adereços (relógios, pulseiras e anéis), molharem as mãos antes de colocar o sabão e acionar o produto evitando a sua contaminação. Em relação à lavagem, são verificados os itens fricção completa (palmas, dorso, interdigitais, polegar, articulações, unhas e punhos) e tempo utilizado comparado ao recomendado (15 segundos). No que se refere ao enxágüe, observou-se se o mesmo era feito no sentido das mãos para os cotovelos e, finalmente, quanto à secagem, se foi utilizado o papel toalha, inclusive como barreira para o fechamento da torneira. Este Check List foi submetido à validação, a fim de verificar a sua clareza e pertinência.

A entrevista e observação foram realizadas a partir de horários convenientes aos profissionais de saúde, inseridos em seus locais de trabalho. Os dados foram organizados em tabelas a partir da descrição das freqüências, assim como de uma análise de freqüência utilizando o Teste-G (Williams). Este teste foi usado para comparar a proporção de profissionais de enfermagem que realizam, segundo as normas do Ministério da Saúde. Todas as análises foram realizadas empregando-se o software Bioestat 2.0 .

\section{RESULTADOS E DISCUSSÃO}

Participaram desta pesquisa profissionais, o que correspondeu a $100 \%$ da 
população atuante nos setor em estudo. Um total de 3 profissionais se recusaram a participar da pesquisa.

A análise descritiva das variáveis sexos, idade, categoria profissional, tempo de formação, tempo de trabalho na maternidade, e número de empregos é descrita na tabela $\mathrm{I}$. $\mathrm{Na}$ análise global, em relação ao sexo observou-se um predomínio do feminino 95,2\%. Nesse sentido, pode-se afirmar que persiste a feminização na enfermagem brasileira, o que pode ser observada tanto na qualificação universitária como nos níveis médio e técnico. ${ }^{16}$

A idade variou entre 19 a 50 anos, Houve predomínio de profissionais na faixa etária de 30 a 40 de 55,5\%. Os entrevistados deste estudo eram: técnicos de enfermagem $74,7 \%$ e enfermeiros $25,3 \%$.

Em relação ao tempo de formação, houve uma maior percentagem de profissionais com tempo de formação entre cinco a nove anos 35\%. Quanto à atuação profissional, entre os $100 \%$ dos profissionais pesquisados, a maioria $51 \%$ tinha menos de 4 anos de experiência no hospital. Esse resultado pode refletir o alto número de contratações.

Observa-se no estudo que a maioria dos profissionais de saúde tem mais de um vínculo empregatício, onde tantos $63,4 \%$ de $100 \%$ afirmaram que possuíam mais de um emprego. Verifica-se que estes profissionais têm mais dificuldades em realizar a prática de lavagem das mãos de forma correta, podendo estar relacionado ao cansaço devido ao excesso de trabalho, comprometimento da capacidade de concentração, falta de tempo para aprimoramento através de cursos de atualização e de leituras (BRASIL, 2011).

Tabela I- Perfil sociodemográfico dos profissionais de enfermagem de uma maternidade do município de Caruaru-PE.

\begin{tabular}{|c|c|c|c|}
\hline \multicolumn{2}{|l|}{ Tabela sociodemográfica } & $\mathrm{N}$ & $\%$ \\
\hline \multirow[t]{3}{*}{ Sexo } & Feminino & 60 & 95,2 \\
\hline & Masculino & 3 & 4,8 \\
\hline & Total & 63 & 100 \\
\hline \multirow[t]{6}{*}{ Idade } & $19-29$ & 10 & 16 \\
\hline & $30-40$ & 35 & 55,5 \\
\hline & $41-51$ & 10 & 16 \\
\hline & $\begin{array}{l}\text { Igual ou acima de } 52 \\
\text { anos }\end{array}$ & 8 & 12,5 \\
\hline & Outras & 0 & 0 \\
\hline & Total & 63 & 100 \\
\hline \multirow[t]{3}{*}{ Categoria profissional } & Enfermeiro & 16 & 25,3 \\
\hline & Tec. Enfermagem & 47 & 74,7 \\
\hline & Total & 63 & 100 \\
\hline \multirow[t]{6}{*}{ Ano de formação } & Maior que 30 & 4 & 6,3 \\
\hline & $29-20$ & 8 & 12,4 \\
\hline & 19 a 10 & 21 & 33,3 \\
\hline & 9 a 5 & 22 & 35 \\
\hline & Menor que 4 & 8 & 13 \\
\hline & Total & 63 & 100 \\
\hline \multirow[t]{6}{*}{$\begin{array}{l}\text { Tempo de trabalho na } \\
\text { maternidade }\end{array}$} & Maior que 30 & 3 & 4,8 \\
\hline & $29-20$ & 3 & 4,8 \\
\hline & 19 a 10 & 8 & 12,4 \\
\hline & 9 a 5 & 17 & 27 \\
\hline & Menor que 4 & 32 & 51 \\
\hline & Total & 63 & 100 \\
\hline \multirow[t]{3}{*}{ Único emprego } & Sim & 13 & 20,6 \\
\hline & Não & 50 & 79,4 \\
\hline & Total & 63 & 100 \\
\hline \multirow[t]{5}{*}{ Total de vínculos empregatícios } & 1 & 14 & 22,3 \\
\hline & 2 & 40 & 63,4 \\
\hline & 3 & 6 & 9,5 \\
\hline & Não aplicável & 3 & 4,8 \\
\hline & Total & 63 & 100 \\
\hline
\end{tabular}


Os Profissionais de Enfermagem foram questionados sobre alguns aspectos de Biossegurança. Na tabela II mostra quais as condutas que os trabalhadores utilizam quando não estão com sujidades aparente nas mãos. É perceptível que a maior parte dos profissionais $(80,7 \%$ dos Técnicos e $87,5 \%$ dos Enfermeiros) aderiu à lavagem das mãos, somente $8,5 \%$ dos Técnicos não lavavam, apenas usavam as luvas de procedimento.

Tabela II - Conhecimento sobre biossegurança de profissionais enfermeiros e técnicos de enfermagem de uma maternidade de Caruaru PE.

\begin{tabular}{|c|c|c|c|c|}
\hline & \multicolumn{2}{|c|}{ Técnicos de enfermagem } & \multicolumn{2}{|c|}{ Enfermeiros } \\
\hline & $\mathrm{N}$ & $\%$ & $\mathrm{~N}$ & $\%$ \\
\hline $\begin{array}{l}\text { Não lava as mãos, mas } \\
\text { fricciona com álcool a } 70 \% \\
\text { após contato com os } \\
\text { pacientes. }\end{array}$ & 1 & 2,2 & 1 & 6,25 \\
\hline $\begin{array}{l}\text { Não lava as mãos, mas } \\
\text { fricciona com álcool a } 70 \% \\
\text { antes do contato com os } \\
\text { pacientes. }\end{array}$ & 1 & 2,2 & 0 & 0 \\
\hline $\begin{array}{l}\text { Não lava as mãos,mas } \\
\text { fricciona com álcool a } 70 \% \\
\text { apenas se for executar } \\
\text { algum procedimento. }\end{array}$ & 0 & 0 & 0 & 0 \\
\hline $\begin{array}{l}\text { Não lava as mãos com água } \\
\text { e sabão e não fricciona } \\
\text { álcool a } 70 \% \text {. }\end{array}$ & 0 & 0 & 0 & 0 \\
\hline $\begin{array}{l}\text { Não lavas as mãos com } \\
\text { água e sabão, mas usa luvas } \\
\text { de procedimento. }\end{array}$ & 4 & 8,5 & 0 & 0 \\
\hline $\begin{array}{l}\text { Não lava as mãos, mas } \\
\text { fricciona com álcool a } 70 \% \\
\text { antes e após contato com os } \\
\text { pacientes. }\end{array}$ & 2 & 4,2 & 1 & 6,25 \\
\hline $\begin{array}{l}\text { Lava as mãos antes e depois } \\
\text { com água e sabão. }\end{array}$ & 38 & 80,7 & 14 & 87,5 \\
\hline Não sabe & 1 & 2,2 & 0 & 0 \\
\hline Não respondeu & 0 & 0 & 0 & 0 \\
\hline Total & 47 & 100 & 16 & 100 \\
\hline
\end{tabular}

A Tabela III apresenta a frequência da lavagem das mãos, antes e após o uso de luvas, pelos profissionais de Enfermagem, onde a ANVISA ressalta que dentre todas as medidas adotadas com o intuito de minimizar os riscos de surtos de infecção, a higienização das mãos sempre teve um papel preponderante (BRASIL, 2011).

No que se refere à adesão da lavagem das mãos antes de calçar as luvas, obteve-se uma maior adesão de profissionais técnicos de enfermagem $63,9 \%$ enquanto a dos profissionais enfermeiros $53,2 \%$ às vezes lavavam, entretanto foi baixo o resultado daqueles que nunca lavavam as mãos tanto para os técnicos $6,4 \%$ quanto para os enfermeiros 6,3\%. Em contrapartida, quando questionados sobre a freqüência da higienização, após a retirada das luvas, apenas $6,4 \%$ dos profissionais técnicos as vezes lavavam, enquanto 93,6\% lavavam sempre, o que representou $100 \%$ dos enfermeiros. Pode-se constatar uma maior adesão por partes dos profissionais após retirar as luvas do que antes do seu uso. É notório que a resistência dos profissionais em realizar a lavagem das mãos, em todas as suas indicações e de maneira correta, é um desafio para os controladores de infecção, apesar da mesma, ser simples e de suma importância. 
Tabela III- Frequência da higienização das mãos dos profissionais antes e após a utilização da luva de procedimento de uma maternidade de Caruaru-PE.

\begin{tabular}{|l|c|c|c|c|}
\hline & \multicolumn{2}{|c|}{ Técnicos de enfermagem } & \multicolumn{2}{c|}{ Enfermeiros } \\
\hline & $\mathrm{N}$ & $\%$ & $\mathrm{~N}$ & $\%$ \\
\hline ANTES & & & & \\
\hline As vezes lava & 14 & 29,7 & 9 & 56,2 \\
\hline Nunca lava & 3 & 6,4 & 1 & 6,3 \\
\hline Sempre lava & 30 & 63,9 & 6 & 37,5 \\
\hline Não sabe & 0 & 0 & 0 & 0 \\
\hline Não respondeu & 0 & 0 & 0 & 0 \\
\hline Total & 47 & 100 & 16 & 100 \\
\hline & & & & \\
\hline APÓS & & & & \\
\hline As vezes lava & 3 & 6,4 & 0 & 0 \\
\hline Nunca lava & 0 & 0 & 0 & 0 \\
\hline Sempre lava & 44 & 93,6 & 16 & 100 \\
\hline Não sabe & 0 & 0 & 0 & 0 \\
\hline Não respondeu & 0 & 0 & 0 & 0 \\
\hline Total & 47 & 100 & 16 & 100 \\
\hline
\end{tabular}

Os técnicos de enfermagem compreendem a maioria dos que participaram da pesquisa e conseqüentemente, representara o maior percentual de profissionais observados. Além disso, estes profissionais permanecem fixos no setor realizando procedimentos invasivos e mantém maior contato com o paciente necessitando realizar mais vezes a técnica da lavagem das mãos.

Conforme as observações realizadas (Tabela IV), os profissionais técnicos de enfermagem respeitaram a ordem seqüencial da técnica de lavagem de mãos. Quanto à fase de preparo, destacou-se a inobservância da retirada de adornos (anéis, relógios e pulseiras) por grande parte dos participantes técnicos de enfermagem (49\%). Vale destacar que de acordo com Scheidt ( 2006), o uso de anéis ou alianças, pelos profissionais de saúde na realização das atividades assistenciais, propicia a permanência da flora transitória sob estes adereços, possibilitando a disseminação de microrganismos patogênicos durante meses.

Ressalta-se que uma grande parte dos profissionais técnicos, não realizaram a fricção completa das mãos, especialmente a fricção das unhas $(59,5 \%)$, conforme recomendam os especialistas. Quanto a fricção do polegar houve diferença estatisticamente significante $\mathrm{p}_{=}(0,0003)$ quando comparados enfermeiros e técnicos.

Quanto à última etapa da técnica, a maioria dos voluntários observados $(57,4 \%)$ não utilizou o papel toalha (usado para a secagem das mãos) como barreira no fechamento da torneira, proporcionando a recontaminação das mãos. Em relação aos enfermeiros observados, nota se que a maioria respeitou a ordem seqüencial da técnica de lavagem de mãos. Visto que tantos (69\%) dos profissionais retiraram seus adornos (anéis, relógios e pulseiras) antes de realizar a técnica.

No que se refere as etapas "molhar as mãos", "utilizar sabão ou álcool gel” e “enxaguar mãos”, 100\% dos enfermeiros conseguiram realizar a técnica corretamente, isto implica a importância de conhecer a pratica correta. A higienização simples das mãos, pode possivelmente remover a microbiota transitória apenas com a utilização de água e sabão e a fricção das mãos, 
possibilitando o controle de infecção hospitalar diante do contato com os pacientes (BRASIL, 2011).

No que se refere à fase denominada "lavagem", todos os profissionais observados utilizaram tempo superior ao recomendado (15 segundos). Certamente, o excesso de tempo deve-se ao fato do procedimento em questão, não ter sido realizado espontaneamente, mas em função de ser alvo do estudo ( ANVISA, 2007).

Tabela IV- Passo da técnica da lavagem das mãos pelos profissionais de enfermagem

\begin{tabular}{|c|c|c|c|c|c|}
\hline \multirow[t]{2}{*}{$\begin{array}{l}\text { PASSO DA } \\
\text { TÉCNICA }\end{array}$} & \multicolumn{3}{|c|}{ Técnicos de enfermagem } & \multicolumn{2}{|c|}{ Enfermeiros } \\
\hline & & $\mathrm{N}$ & $\%$ & $\mathrm{~N}$ & $\%$ \\
\hline \multirow[t]{4}{*}{$\begin{array}{l}\text { Retirou jóias, pulseiras } \\
\text { e relógios }\end{array}$} & Sim & 20 & 42,5 & 11 & 68,5 \\
\hline & Não & 23 & 49 & 3 & 18,75 \\
\hline & Parcialmente & 4 & 4,2 & 2 & 12,75 \\
\hline & Total & 47 & 100 & 16 & 100 \\
\hline \multirow[t]{4}{*}{ Molhou as mãos } & Sim & 47 & 100 & 16 & 100 \\
\hline & & 0 & 0 & 0 & 0 \\
\hline & Parcialmente & 0 & 0 & 0 & 0 \\
\hline & Total & 47 & 100 & 16 & 100 \\
\hline \multirow[t]{4}{*}{$\begin{array}{l}\text { Utilizou sabão ou } \\
\text { álcool gel }\end{array}$} & Sim & 46 & 98 & 16 & 100 \\
\hline & Não & 1 & 2,1 & 0 & 0 \\
\hline & Parcialmente & 0 & 0 & 0 & 0 \\
\hline & Total & 47 & 100 & 16 & 100 \\
\hline \multirow[t]{4}{*}{$\begin{array}{l}\text { Esfregou palma a } \\
\text { palma }\end{array}$} & Sim & 45 & 96 & 15 & 93,7 \\
\hline & Não & 2 & 4,2 & 1 & 6,3 \\
\hline & Parcialmente & 0 & 0 & 0 & 0 \\
\hline & Total & 47 & 100 & 16 & 100 \\
\hline \multirow[t]{4}{*}{$\begin{array}{l}\text { Esfregou palma com } \\
\text { dorso }\end{array}$} & Sim & 40 & 85,1 & 15 & 93,7 \\
\hline & Não & 3 & 6,3 & 1 & 6,3 \\
\hline & Parcialmente & 4 & 8,5 & 0 & 0 \\
\hline & Total & 47 & 100 & 16 & 100 \\
\hline
\end{tabular}

\begin{tabular}{|c|c|c|c|c|c|}
\hline \multirow[t]{4}{*}{$\begin{array}{l}\text { Esfregou espaços } \\
\text { interdigitais }\end{array}$} & Sim & 31 & 66 & 11 & 68,5 \\
\hline & Não & 15 & 32 & 5 & 31,5 \\
\hline & Parcialmente & 1 & 2,1 & 0 & 0 \\
\hline & Total & 47 & 100 & 16 & 100 \\
\hline \multirow[t]{4}{*}{ Esfregou polegar } & Sim & 25 & 83,1 & 16 & 100 \\
\hline & Não & 20 & 42,5 & 0 & 0 \\
\hline & Parcialmente & 2 & 4,2 & 0 & 0 \\
\hline & Total & 47 & 100 & 16 & 100 \\
\hline \multirow[t]{4}{*}{ Esfregou unhas } & Sim & 18 & 38,2 & 11 & 68,5 \\
\hline & Não & 28 & 59,5 & 5 & 31,5 \\
\hline & Parcialmente & 1 & 2,1 & 0 & 0 \\
\hline & Total & 47 & 100 & 16 & 100 \\
\hline \multirow[t]{4}{*}{ Esfregou punhos } & Sim & 29 & 62 & 13 & 81,2 \\
\hline & Não & 17 & 36,1 & 2 & 12,5 \\
\hline & Parcialmente & 1 & 2,1 & 1 & 6,3 \\
\hline & Total & 47 & 100 & 16 & 100 \\
\hline \multirow[t]{4}{*}{ Enxaguou mãos } & Sim & 46 & 97,2 & 16 & 100 \\
\hline & Não & 1 & 2,1 & 0 & 0 \\
\hline & Parcialmente & 0 & 0 & 0 & 0 \\
\hline & Total & 47 & 100 & 16 & 100 \\
\hline \multirow[t]{4}{*}{ Utilizou papel toalha } & Sim & 46 & 97,2 & 15 & 93,7 \\
\hline & Não & 1 & 2,1 & 1 & 6,3 \\
\hline & Parcialmente & 0 & 0 & 0 & 0 \\
\hline & Total & 47 & 100 & 16 & 100 \\
\hline \multirow[t]{4}{*}{$\begin{array}{l}\text { Fechou a torneira com } \\
\text { papel }\end{array}$} & Sim & 20 & 45,5 & 11 & 68,5 \\
\hline & Não & 27 & 57,4 & 5 & 31,5 \\
\hline & Parcialmente & 0 & 0 & 0 & 0 \\
\hline & Total & 47 & 100 & 16 & 100 \\
\hline
\end{tabular}

*Para determinar se havia diferença estatisticamente significante entre os profissionais de enfermagem nos itens que compõem a técnica de lavagem das mãos, foi utilizado o Teste-G (Williams). 


\section{CONCLUSÃO}

Quanto à comparação entre os enfermeiros e os técnicos em enfermagem em relação à execução da técnica da lavagem das mãos em cada passo conclui - se que os profissionais enfermeiros em relação aos técnicos apresentaram um melhor desempenho na realização da seqüência correta da técnica.

Embora a maternidade disponha dos equipamentos e produtos necessários para a lavagem das mãos e da existência de cartazes mostrando todas as etapas do processo desta higienização, a maioria dos profissionais principalmente os técnicos segundo resultado não realizam a sequência conforme a técnica correta. Sendo esta um procedimento imprescindível desenvolvido pelos profissionais de saúde no combate às infecções hospitalares. Visto a necessidade de conscientizar os profissionais para obter uma maior adesão a essa rotina na prevenção das IH, uma vez que a intervenção somente educacional tem sido insuficiente no que diz respeito à forma correta de lavagem das mãos.

\section{REFERÊNCIAS}

1. BURTON, G; ENGELKIRK, P. Microbiologia para as Ciências da Saúde. Ed: Guanabara Koogan; 2005.

2. BRASIL. Segurança do paciente: higienização das mãos. Brasília, DF.
(2007)98 p. disponível:

www.anvisa.gov.br/servicosaude/man uais/paciente hig_maos.pdf Acesso em: 24 agosto. 2011.

3. BRASIL. Manual de higienização das mãos em serviços de saúde/ Agência Nacional de Vigilância Sanitária. Brasília: Anvisa. 2007; 52 p.

4. CARRARO, T; KNOBE, R; FRELLO, A; GREGÓRIO, V; GRÜDTNER, D; RADÜNZ, V; et al. O papel da equipe de saúde no cuidado e conforto no trabalho de parto e parto: opinião de puerperas. Texto Contexto Enferm.2008; vol.17, (n.3), pp. 502509.

\section{CONSELHO NACIONAL DE}

SAUDE. Diretrizes e normas regulamentadoras de pesquisas envolvendo seres humanos. Pesqui. Odontol. Bras. 2003; vol.17, p. 33-41.

6. FELIX, C; MIYADAHIRA, A. Avaliação da técnica de lavagem das mãos executada por alunos do Curso de Graduação em Enfermagem. Rev. Esc. Enferm. USP. vol.43, n.1, 2009. pp. 139-145.

7. LOPES, M.J.M; LEAL, Sandra M.C. A feminização persistente na qualificação profissional da enfermagem brasileira. Cadernos pagu. 2005;(n.24), pp. 105-125.

8. MARTINEZ, M. R; CAMPOS, L.A, NOGUEIRA, P.C. Adesão à técnica de lavagem de mãos em Unidade de Terapia Intensiva Neonatal. Rev. paul. pediatr. 2009; vol.27, (n.2), pp. 179185.

9. MARTINI, A.C. Lavagem das mãos no olhar de trabalhadores de enfermagem [dissertation]. Porto Alegre: Universidade do Rio Grande do Sul; 2004.117 p. 
10. PAULA, D.M; OLIVEIRA, A.C; CARDOSO, C.S. Precauções de contato: conhecimento e comportamento dos profissionais de um centro de terapia intensiva em um hospital geral de Belo Horizonte [dissertation]. Belo Horizonte: Universidade Federal de Minas Gerais/UFMG; 2008. 106 p.

11. PALOS, M.A.P; SILVA, SILVA V.B.D; GIR, E; CANINI, S.R.M; ANDERS, P.S; LEÃO, L.S.N.

Microbiota das mãos de mães e profissionais de saúde de uma maternidade de Goiânia. Revista eletrônica de enfermagem. Goiânia, 2009. Acesso em: http://www.fen.ufg.br/revista/v11/n3/v 11n3a14.htm, disponível em: 22/08/2010.

12. PEREIRA, M.S; SOUZA, A.C.S; TIPPLE, A.F.V; PRADO, M.A. A infecção hospitalar e suas implicações para o cuidar da enfermagem. Texto contexto enferm. 2005; vol.14 (no.2): pp. 250-257.

13. SANTOS, F; GONÇALVES, V. Lavagem das mãos no controle da infecção hospitalar: um estudo sobre a execução da técnica. Revista Enfermagem Integrada - Ipatinga: Unileste. 2009; V.2, (N.1), PP. 152163.

14. SCHEIDT, K; CARVALHO, M. Avaliação prática da lavagem das mãos pelos profissionais de saúde em atividades lúdico-educativas. $\mathrm{R}$ Enferm UERJ. 2006 abr/jun; 14 (2):221-5.

15. SMELTZER, Suzanne C. et al. BRUNNER \& SUDDARTH: Tratado de Enfermagem Médico-Cirúrgica. 10a ed. 2011.

16. VERONESI, R. Tratado de infectologia. Ed: Atheneu; 2005.

17. XIMENES N.F.C; ARAGÃO, A.E.A; PONTE, M.A.C; MONTEIRO, M.A.A. Biossegurança na assistência ao parto: uma análise dos saberes dos acadêmicos de enfermagem. Enfermería Global. 2007; ( $\left.\mathrm{N}^{\circ} 11\right)$,p. 11. 